\title{
Aspergillus rhinosinusitis with ethmoid cell involvement in a patient with acute myeloblastic leukemia
}

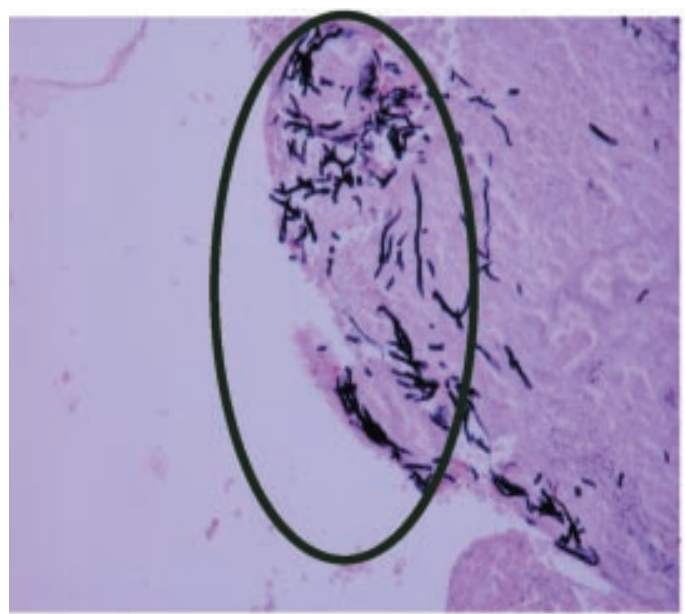

Fig. 1

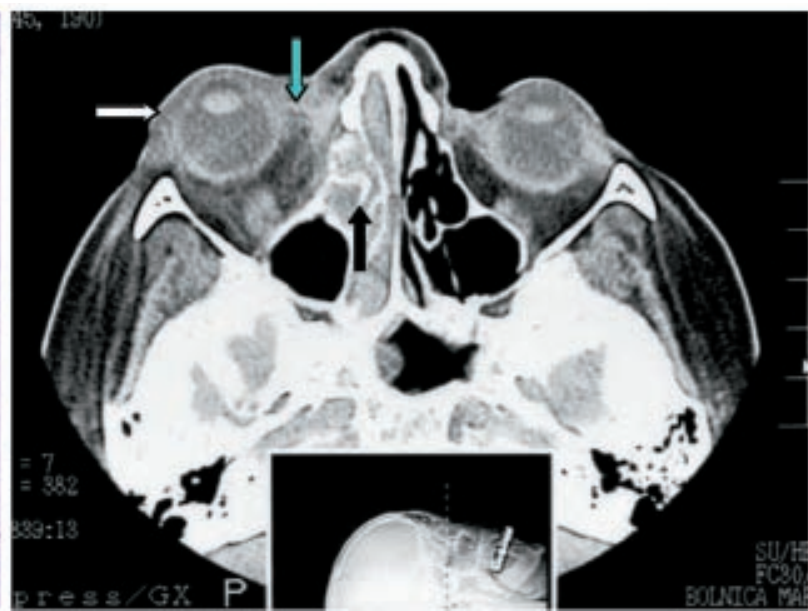

Fig. 2

A 58-year-old female patient with chemotherapy-resistant acute myeloblastic leukemia had noticed a swelling of the left side of her face, accompanied by pain in the left half of the upper jaw. A few days later a nasal discharge appeared, she became febrile, her right eye was swollen, red, exophthalmos and diplopia were evident. Sinography revealed a shadowing of the right maxillary sinus while rhinoscopy exposed a necrotic mucosa in the middle nasal concha. Histologic examination of the nasal mucosa was performed: hematoxylin-eosin staining showed presence of fungi in the necrotic nasal mucosa, with evident dichotomous branching and septation of fungal hyphae, while silver staining acc. to Groccott clearly identified the fungi (Fig. 1). CT showed, that right ethmoidal sinus cells were filled with masses (black arrow), and a destruction of the orbital wall and penetration of masses into the orbit (blue arrow) was visible. Present was exophthalmos of the right eyeball (white arrow) and a partly swollen mucosa of the sphenoidal sinus (Fig. 2). The patient was treated with liposomal amphotericin B and repeated endoscopic ethmoidectomy. The disease was stabilized for seven months, when she was admitted again with same signs and despite rapid intervention died soon afterwards.

Conclusion: Only rare cases of Aspergillus ethmoiditis are described in the literature [1]. Its onset is quite unspecific, the mortality rate extremely high, so early identification of the infection is important [2]. The only suitable diagnostic investigation is histologic verification of fungi [3]. Treatment consists of medical and surgical intervention.

\section{Marjana Glaser, Bojan Lanišnik, and Kristina Gornik-Kramberger}

\section{References}

1. Min YG, Kim HS, Lee KS, Kang MK, Han MH (1996) Aspergillus sinusitis: clinical aspects and treatment outcomes. Otorhinolaryngol Head Neck Surg 115: 49-52

2. Kim DG, Hong SC, Kim HJ, Chi JG, Han MH, Choi KS, Han DH (1993) Cerebral aspergillosis in immunologically competent patient. Surg Neurol 40: 326-331

3. Ascioglu S, de Pauw BE, Meis JFGM (2000) Prophylaxis and treatment of fungal infections associated with haematogical malignancies. Int J Antimicrob Agents 16: 159-168

4. Bunc G, Vorsic M (2001) Long-term survival following treatment of multiple supra- and infratentorial aspergillus brain abscesses. Wien Klin Wochenschr 113 [Suppl 3]: 69-74

Key words: Aspergillus rhinosinusitis, ethmoid cells, acute leukemia.

Correspondence: Marjana Glaser, M.D., Ph.D., Department of Hematology, University Hospital Maribor, Ljubljanska 5, 2000 Maribor, Slovenia, E-mail: m.glaser@sb-mb.si 